\title{
Are Absorbent Pads Suitable Alternatives for Tattoo Dressing?
}

\author{
Yeang-Bin Pan ${ }^{1}$, Helen Heacock ${ }^{2}$, Fred Shaw ${ }^{3}$ \\ 1 Lead Author, School of Health Sciences, British Columbia Institute of Technology, Burnaby, BC, 3700 Willingdon Ave, Burnaby, BC V5G \\ $3 \mathrm{H} 2$ \\ 2 Supervisor, School of Health Sciences, British Columbia Institute of Technology, Burnaby, BC, 3700 Willingdon Ave, Burnaby, BC V5G 3H2 \\ 3 Contributor, School of Health Sciences, British Columbia Institute of Technology, Burnaby, BC, 3700 Willingdon Ave, Burnaby, BC V5G \\ $3 \mathrm{H} 2$
}

\begin{abstract}
Background: Tattoo is a form of body modification that involves injecting ink underneath the epidermis using a needle. According to BC's Guidelines for Personal Services Establishments this kind of invasive procedure will require wound dressing but the kind and sterility of the wound dressing has not been specified. During a tattoo convention an Environmental Health Officer (EHO) noticed that some tattoo artists were using absorbent pads for wound dressing. Absorbent pads are not sterile; therefore there is a concern of microbial infections at the tattoo wounds. This study examined the viability of using absorbent pads, which are non-sterile, as tattoo wound dressings.
\end{abstract}

Method: 3M Quick Swabs were used to swab absorbent pads to collect the microorganisms present on the surface. 3M Petrifilm E. coli/Coliform Count Plates were used to culture E. coli and Coliform which could be present on the Saniderm ${ }^{\mathrm{TM}}$ sterile tattoo dressing and absorbent pads. The presence of $E$. coli colonies on the petrifilm would show up as blue dots, while coliform colonies would be red dots. The total colony forming units (CFU), which include both E. coli and coliform colonies, were counted to assess the general sanitation conditions of both types of dressing.

Results: Zero CFU were found from sampling the Saniderm ${ }^{\mathrm{TM}}$ dressings and absorbent pads purchased from tattoo supply shops within Metro Vancouver.

Conclusions: In the field of Environmental Health, coliform is often used as an indicator for the degree of sanitary quality; while E. coli is used as an indicator for fecal contamination.

Therefore, the results of this study, indicated that the absorbent pads have high degree sanitation and are free from fecal contamination.

Keywords: Absorbent pads, meat pads, tattoo, dressing, wound dressing, sterile dressing

\section{Introduction}

Absorbent pads (AP) are generally used by the food industry as part of their raw meat, fish or poultry packaging to absorb liquid exuded from food products.(1) These pads are generally white in colour and are found under the food products, at the base of styrofoam trays. Some of these AP may have antimicrobial properties of sachets, which contain antimicrobial compounds that may kill bacteria or inhibit their growth.(1, 2) Despite this antimicrobial property, the AP themselves are not intrinsically sterile, not meant to be used for wound care, and not known to be antimicrobial on blood borne pathogens, which are usually associated with tattooing related infections. These uncertainties are why Environmental Health Officers (EHOs) are concerned about 
tattoo artists using APs as non-sterile wound dressings for their customers. This problem was reported by an EHO. While doing an inspection at a tattoo tradeshow, the EHO noticed people walking around with APs bandaged to their arms and some of these people also had blood trickling down to their forearm from the APs area. The EHO found out that some tattoo artists were doing tattoos at the tradeshow and were using APs as dressing on their customers' new tattoos. According to section 4.4.2 Client of the BC's Guidelines for Personal Services Establishments: "after an invasive procedure the operator is to provide the client with appropriate protective equipment and garments, such as eye protection or coverings for clothing".(3) The definition of "appropriate protective equipment and garments" is not clearly prescribed in the guidelines; therefore, the EHO could not declare the use of APs as non-sterile dressing as a health hazard. This evidence review aims to find out if absorbent pads used as non-sterile dressings for tattoo wound care, pose a significant public health risk when compared to using sterile dressings. The review will incorporate other similar research on the topic of sterile vs. non-sterile cases to determine the suitability of using absorbent pads for food products as dressings for tattoo wound care.

\section{Evidence Review Regulations and Guidelines}

Under section 4.4.2 Client of the

BC's Guidelines for Personal Services Establishments (PSE), the definition of "appropriate protective equipment and garments" is not clearly prescribed.(3) Sometimes regulations and guidelines use vague descriptions to give enforcement officers and/or business operators more latitude in enforcement and compliance options respectively. However, in this case more specific guidance on the type of dressing to use would better protect PSE customers from infections and be easier for the officers to enforce.(4) For example "sterile protective equipment and garments" or "sterile or non-sterile and antimicrobial protective equipment and garments".

\section{Blood Borne Illness}

Without adequate dressing for open wounds it is easier for diseases such as HIV, Hepatitis B and C viruses to spread within the population.(5) These blood borne diseases can be spread when infected blood enters a person's broken skin, wounds, or mucous membranes. The inadequate absorbency of the AP is a potential public health risk for others who come in contact with newly tattooed customers. This risk can be easily mitigated if the type of dressing is more clearly specified in the PSE guidelines.

\section{The Absorbency of Absorbent Pad}

The absorbent pad is part of food product packaging. It absorbs exuded liquid to make the food product look more appealing. Despite being inherently absorbent, APs do have their limitation in absorbency. When squeezed by enough force, APs will expel the liquid they had already absorbed. (1) The trickling of the blood from the APs observed by the EHO might be due to the regular flexing of the arm muscles, which forced the absorbed blood out from the APs, or it could mean that the AP was bandaged on too tightly, thus unable to absorb the seeping blood from the tattoo wound, or both. Moreover, APs are meant to be put on flat surfaces of a Styrofoam tray and do not have much elasticity to form smoothly around curved surfaces, such as the contour of a human arm. This "bad fit" and gravity could also be the reasons why APs could not adequately absorb blood flowing from a wound on a non-flat surface.

\section{The Sachet's Antimicrobial Properties}

Sachet is a kind of pad which has antimicrobial compounds incorporated into 
it. (1) However, by using sachets to dress wounds, the antimicrobial compounds they contain might not be effective in preventing blood borne illness that are associated with tattooing. This is because the sachet was designed to be part of food packaging; therefore, antimicrobial additives should be tailored to fight food borne diseases and not blood borne ones.

\section{Cost of Sterile Dressings}

The type of wound, the nature by which it was inflicted, its location and severity, will all affect type of dressing needed. Depending on the type, the cost of dressing required could range from one to fifty-five dollars per week. (6) In another study, with comparable size, the cost of a sterile dressing was about twenty-five times more than a non-sterile dressing. (7) For tattoo parlors with potentially hundreds of customers per year, using inexpensive nonsterile dressings like APs, can keep their cost down significantly; thus it would be difficult to have the cooperation from tattoo parlor owners and to be able to bring the cases to court, if we cannot prove that using AP as tattoo dressing is a health hazard.

\section{Sterile vs. Non-sterile}

\section{Gloves}

In this area, there are more studies done in regards to gloves than dressings. All three studies concluded that for certain medical procedures, sterile gloves are totally acceptable from the clinical standpoint. (810) The first study is about minor skin excisions, which is very similar to tattoos in terms of the severity of the trauma inflicted on the skin. (8) The other study took palmar swab samples from three categories of gloves: self-donned non-sterile, self-donned and assisted-donned sterile, from volunteers.

(9) The bacterial load found from each categories was statistically significant but was concluded to be clinically irrelevant. (9) This experiment does help demonstrate that the ways of putting on gloves have a significant impact on gloves' sterility (post contamination). The last study "demonstrated that there is no clinically important difference in infection rates between using clean nonsterile gloves and sterile gloves during the repair of uncomplicated traumatic lacerations". (10) This confirms the previous conclusion of bacterial contamination from putting on gloves having no clinical significance.

\section{Clean Dressing}

The study compared sterile gauze with panty liners, sanitary napkins, diapers and Coban (3M, self-adherent wrap). It found that $2 / 20$ sterile gauze, $1 / 20$ panty liners, $0 / 20$ sanitary napkins, $15 / 20$ diapers and 2/5 Coban had bacteria of interest growing on them. (7) If we also factor in the cost of these "dressings", one can conclude that using sterile gauze is not the best nor the most cost effective way of dressing a wound. (7) If we were to eliminate Coban, which is meant to secure dressings, and diapers, which are meant to hold feces and urine, then we can conclude that sterile gauze is actually the most contaminated in comparison with the rest of the dressings. If we assume there were no problems with the sterilization process of the gauze, what other factors could contribute to this?

\section{Importance of Being Sterile}

A case of woman's surgical wound becoming infected from using clean, nonsterile gloves and sanitary napkins was reported. (13) However, in a previous study, sanitary napkins were found to be free of bacteria. (7) Another study found that three separate cases got serious infections from using non-sterile crepe roller bandages. (14) So contrary to other studies, these are real life incidents of possible infections from using non-sterile dressings. Therefore, more research is needed to determine the 
suitability of using non-sterile dressing for wound care.

\section{Dispensing and Handling}

A study showed that non-sterile disposable gloves used in a hospital became more pathogen contaminated as time passed. (11) Moreover, another article describes the winning glove dispenser design, which aims to reduce contamination and by minimizing physical contact between people and gloves. (12) These articles raised the questions of what other possibility besides the intrinsic characteristics of the absorbent pads could cause contamination.

\section{Conclusion}

From conducting the evidence review, there is conflicting information on whether non-sterile is a suitable low-cost alternative for wound care or not. From a logical, cost effective perspective, using a clean, non-sterile dressing should not be a major problem. While on the other hand, the real life cases of infection from using such dressing cannot be lightly dismissed. The purpose of this research project was to determine if absorbent pads are a suitable alternative for tattoo wound care from the public health perspective.

\section{Methods \& Materials Introduction}

The study involved sampling and culturing of 20 Saniderm $^{\mathrm{TM}} 10 \mathrm{~cm}$ by 20.3 $\mathrm{cm}$ sterile dressing and 30 absorbent pads ordered directly from tattoo supply shops in Metro Vancouver. Saniderm ${ }^{\mathrm{TM}}$ dressings were used as control sample, while absorbent pads were the experiment samples. The culturing of all samples was performed in at the laboratory at BCIT Burnaby Campus. The procedures were adapted from BC CDC's Environmental Hygiene Monitoring: A Guide for Environmental Health Officers and product instructions for the $3 \mathrm{M}$ products used. (1517)

\begin{abstract}
Materials
The materials used in this research study included fifty $3 \mathrm{M}$ Quick Swabs, used for sampling the surfaces of absorbent pads and Saniderm $^{\mathrm{TM}}$, fifty $3 \mathrm{M}$ Petrifilm $E$. coli/Coliform Count Plates, used for culturing E. coli and Coliform, one 3M Petrifilm Spreader, used to evenly spread out the letheen broth from the 3M Quick Swabs on Petrifilms, one $10 \mathrm{~cm}^{2}$ stencil, used to set swabbing area, thirty $11.4 \mathrm{~cm}$ by $17.8 \mathrm{~cm}$ Absorbent Pads, a roll of $10 \mathrm{~cm}$ by $20.3 \mathrm{~cm}$ yards Saniderm ${ }^{\mathrm{TM}}$, disposal gloves and incubator.
\end{abstract}

\section{Methods}

Firstly, the absorbent pads and Saniderm ${ }^{\mathrm{TM}}$ sterile dressing were swabbed using 3M Quick Swabs by following the sampling protocol of the BC CDC's Environmental Hygiene Monitoring Guide. $(15,16)$ Secondly, the letheen broth from the 3M Quick Swabs were transferred on 3M Petrifilm E. coli/Coliform Count Plates by using the Interpretation Guide for $3 \mathrm{M}$ Petrifilm E. coli/Coliform Count Plates and Wet Swabbing Method from the 3M Quick Swab Product Instructions. $(16,17)$ Thirdly, the count plates were incubated as according to the AOAC Official Method 991.14 Coliform and Escherichia coli Counts in Foods Dry Rehydratable Film for twentyfour hours. (18) Finally, the 3M Petrifilm $E$. coli/Coliform Count Plate Interpretation Guide was used for the enumeration of viable $E$. coli and Coliform colonies found on the Petrifilms. (17)

\section{Results}

The results of sampling the Saniderm ${ }^{\mathrm{TM}}$ dressings and absorbent pads purchased from tattoo supply shops within Metro Vancouver are shown in Table I. Coliform is often used as an indicator for the degree of sanitary quality; while E. coli is used as an indicator for fecal contamination. (19) There was zero coliform and E. coli 
colony-forming units found in all the sampled Saniderm ${ }^{\mathrm{TM}}$ dressings and absorbent pads, which indicated that the absorbent pads have high degree sanitation and are free from fecal contamination.

\section{Discussion}

The absence of coliform and E. coli CFU from absorbent pads is a positive finding for tattoo shops, which are using absorbent pads as tattoo wound dressing, and tattoo customers, whose tattoo wounds were dressed with absorbent pads. The results suggests that the absorbent pads sold by the tattoo supply shops in the Metro Vancouver area are generally sanitary and free from fecal contamination. From this result we can extrapolate this as a possible reason for why despite some tattoo shops using absorbent pads as tattoo dressings, there has not been a reported outbreak associated with their use within Metro Vancouver.

The results from this study also agrees with previous research about sterile and non-sterile dressings. (7) Under usual circumstances, the differences between the two types of dressing are marginal when used for shallow wounds such as tattoos.

However, the absence of coliform and E. coli bacteria could be due to the prolonged lack of water and nutrients, which lowered the survival rate significantly. (20) However, the absence of coliform and $E$. coli $\mathrm{CFU}$ is not proof that absorbent pads are totally free from biological contaminations, as there could be other type of pathogens present on the pads which were not tested for. One of such pathogens could be Salmonella spp., which could survive in a low-moisture environment for extended periods of time and is also a common food borne disease. (21)

\section{Limitations}

Due to funding constraints, it is not possible to test the Saniderm ${ }^{\mathrm{TM}}$ and absorbent pads for other possible human pathogens. In order to test for these types of pathogens, the samples need to be tested in a professional biological laboratory, which would be very costly. Moreover, it is better to simulate "real life" situations (i.e. using the absorbent pad samples from tattoo shops which are using them; in comparison to just using ones purchased straight from a tattoo supply shop). This could be achieved through collection of absorbents from tattoo shops that are using them. However, this would not be simple because not all tattoo shops use absorbent pads and some might not admit this to a researcher who they have no reason to trust. In addition, few tattoo shops are willing to give permission to sample the absorbent pads they are using for fear of liability issues which could arise from the study's results.

\section{Knowledge Translation}

There are four reasons that makes it next to impossible to request tattoo shop operators to use sterile dressing instead of absorbent pads for tattoo dressing. Firstly, from an EHO's perspective, it is currently impossible to prove beyond a reasonable doubt that the use of absorbent pads for tattoo wounds would cause a health hazard. Secondly, in section 4.4.2 Client of the BC's Guidelines for Personal Services

Establishments the definition of "appropriate protective equipment and garments" is not clearly prescribed. (3) Thirdly, results of this study indicated an absence of coliform and E. coli on absorbent pads which generally meant that the pads were in reasonably sanitary condition. Therefore, this study recommend that EHOs continue to allow the use of absorbent pads for tattoo wound dressing until more conclusive studies can be performed to prove otherwise. Lastly, from the monetary perspective, it would be difficult to discourage tattoo shops to use 
sterile dressing over absorbent pads because Saniderm ${ }^{\mathrm{TM}}$ costs ten times as much as absorbent pads in Metro Vancouver area. For a tattoo shop operator to switch from such low cost absorbent pads would require regulatory enforcement, which is currently non-existent.

Despite the above, this study would recommend EHOs to focus their efforts on ensuring proper handling and storing of the absorbent pads in tattoo shops to prevent any contaminations post purchasing absorbent pads from tattoo supply shops.

\section{Future Research}

- Sampling absorbent pads to test for other human pathogens of interest

- Sampling absorbent pads collected from tattoo shops for pathogens or coliform

- Sampling of other type of sterile dressings used for tattoo wounds for pathogens or coliform

\section{Conclusions}

The findings from this study correlated with BC's Guidelines for Personal Services Establishments' undefined "appropriate protective equipment and garments" for wound dressing. This unclear prescription in the guideline allows for tattoo shop operators to use non-sterile and alternative dressings for tattoo wound care on their customers. Despite this, this study showed that if absorbent pads are handled and stored properly, bacteria such as coliform and $E$. coli would be unlikely to survive or propagate on their surfaces.

Although the absence of coliform and $E$. coli does not mean that other type of human pathogens are also absent, but it does indicate that absorbent pads could be maintained in a generally sanitary condition. Therefore, EHOs should focus their efforts on ensuring that tattoo shop operators follow proper hand hygiene protocol and keep the premise in good sanitary condition, which can help prevent contamination of absorbent pads.

\section{Acknowledgements}

This research study was only possible with the help of Dr. Helen Heacock and Fred Shaw. Their tireless support, technical expertise and insights were invaluable to the study's success.

\section{Competing Interests}

The authors declare that they have no competing interests.

\section{Reference}

1. Otoni CG, Espitia PJP, AvenaBustillos RJ, McHugh TH. Trends in antimicrobial food packaging systems: Emitting sachets and absorbent pads. Food Res Int [Internet]. Elsevier Ltd; 2016;83:6073. Available from: http://dx.doi.org/10.1016/j.foodres.20 16.02.018

2. Appendini P, Hotchkiss JH. Review of antimicrobial food packaging. Innov Food Sci Emerg Technol. 2002;3(2):113-26.

3. Branch HP, Health MOF. Guidelines for Personal Service Establishments. 2013;(November).

4. Armstrong ML. Tattooing, body piercing, and permanent cosmetics: a historical and current view of state regulations, with continuing concerns. J Environ Health [Internet]. 2005;67(8):38-43, 54, 53. Available from:

http://www.ncbi.nlm.nih.gov/pubmed $/ 15856663$

5. BC Centre for Disease Control. Communicable Disease Control: 
Blood and Body Fluid Exposure Management. 2010;(August):1-26. Available from: http://www.bccdc.ca/NR/rdonlyres/E 6AF842F-A899-477F-BC905BFF6A4280F6/0/EPI_Guideline_B BF_20100723.pdf.

6. Fonder MA, Lazarus GS, Cowan DA, Aronson-Cook B, Kohli AR, Mamelak AJ. Treating the chronic wound: A practical approach to the care of nonhealing wounds and wound care dressings. J Am Acad Dermatol. 2008;58(2):185-206.

7. Alqahtani M, Lalonde DH. Sterile versus nonsterile clean dressings. Can J Plast Surg [Internet]. 2006;14(1):25-7. Available from: http://www.pubmedcentral.nih.gov/ar ticlerender.fcgi?artid $=2539027 \&$ tool $=$ pmcentrez\&rendertype $=$ abstract

8. Changer P, Case I, Summary S. Sterile or non-sterile gloves for minor skin excisions? 2015;64(11):723-5.

9. Creamer J, Davis K, Rice W. Sterile gloves: Do they make a difference? Am J Surg [Internet]. Elsevier Inc.; 2012;204(6):976-80. Available from: http://dx.doi.org/10.1016/j.amjsurg.20 12.06.003

10. Perelman VS, Francis GJ, Rutledge T, Foote J, Martino F, Dranitsaris G. Sterile Versus Nonsterile Gloves for Repair of Uncomplicated Lacerations in the Emergency Department: A Randomized Controlled Trial. Ann Emerg Med. 2004;43(3):362-70.

11. Karch BAM, Karch FE, Sterile C. w Practice Errors. 2001;101(4):2001.
12. Shakoor S, Jabeen K, Idrees R, Jamil B, Irfan S, Zafar A. Necrotising fasciitis due to Absidia corymbifera in wounds dressed with non sterile bandages. Int Wound J. 2011;8(6):651-5.

13. Hughes KA, Cornwall J, Theis JC, Brooks HJL. Bacterial contamination of unused, disposable non-sterile gloves on a hospital orthopaedic ward. Australas Med J. 2013;6(6):331-8.

14. Amos JR, Moy AS, Gomez A. Design of a new non-sterile glove-dispensing unit to reduce touch-based contamination. Australas Med J. 2014;7(3):171-4.

15. Food Quality Check Program Microbiological Recommendations [Internet]. BC CDC; 2011 [cited 2016Nov21]. Available from: http://www.bccdc.ca/resourcegallery/Documents/Guidelines and Forms/Forms/Labs/PHMRL_FoodQu alityMicroRecommendations2012_16 Dec2011.pdf

16. Quick Swab Product Instructions [Internet]. 3M; 2014 [cited 2016Nov22]. Available from: http://multimedia.3m.com/mws/media /1030809O/quick-swab-productinstructions.pdf

17. E. coli /Coliform Count Interpretation Guide [Internet]. 3M; 2014 [cited 2016Nov22]. Available from: http://multimedia.3m.com/mws/media /236246O/petrifilm-ecoli-coliforminterpretation-guide.pdf

18. AOAC Official Method 991.14 Coliform and Escherichia coli Counts 
in Foods Dry Rehydratable Film (Petrifilmä E. coli/Coliform Count Plateä and Petrifilmä Coliform Count Plateä) Methods.

19. Ashbolt NJ, Grabow WOK, Snozzi $\mathrm{M}$. Indicators of microbial water quality. Indicators of microbial water quality [Internet]. 2001; Available from:

http://www.who.int/water_sanitation_ health/dwq/iwachap13.pdf

20. Albergotti R, Bergeron E. How long do microbes like bacteria and viruses live on surfaces in the home at normal room temperatures? [Internet]. Popular Science. 2002 [cited 2017Mar10]. Available from: How long do microbes like bacteria and viruses live on surfaces in the home at normal room temperatures?

http://www.popsci.com/scitech/article /2002-08/how-long-do-microbesbacteria-and-viruses-live-surfaceshome-normal-room-tem

21. Finn S, Condell O, McClure P, Amezquita A, Fanning S. Mechanisms of survival, responses and sources of Salmonella in lowmoisture environments. 2013Nov14; Available from: https://www.ncbi.nlm.nih.gov/pmc/art icles/PMC3827549/ 